\title{
Zur Kenntniss des Aurins
}

\author{
von \\ J. Herzig und Th. v. Smoluchowski.
}

Aus dem I. chemischen Laboratorium der $k$. $k$. Universität in Wien.

(Vorgelegt in der Sitzung am 11. Jänner 1894.)

Schon vor längerer Zeit hat der Eine von uns ' darauf aufmerksam gemacht, dass das Acetylaurin, sowie die Acetylrosolsäure einer erneuerten gründlichen Bearbeitung bedürfen, da die hiefür geltenden Constitutionsformeln sehr unwahrscheinlich sind. Diese Verbindungen werden als Additionsproducte von Essigsäure-Anhydrid und Aurin, respective Rosolsäure betrachtet, und es ist wohl nicht gut denkbar, dass bei einer Acetylirung eine Addition von Anhydrid stattfinde, ohne dass die beiden vorhandenen Hydroxylwasserstoffe ersetzt werden. Das genaue Studium dieser Verbindungen, welches darauf hin unternommen wurde, ergab zwar ein analytisch ganz klares Resultat, aber die Erklärung desselben im Sinne der jetzt herrschenden Auffassung von der Constitution des Aurins bietet grosse Schwierigkeiten, und zwar schon desshalb, weil die Frage nach der Configuration dieser Körper in naher Beziehung steht zu den Problemen, welche in der Rosanilin- und Auringruppe zu den schwierigsten gehören, und deren Discussion noch jetzt nicht ganz abgeschlossen ist. Obwohl wir die Constitution der Acetylderivate noch nicht ganz sicher ermittelt haben, wollen wir doch unsere bisherigen Resultate hiemit bekanntgeben, da seit der oben citirten Publication schon einige Zeit verstrichen ist und wir uns dieses Arbeitsgebiet sichern möchten.

1 Monatshefte für Chemie, XIII, S. 422. 
Das Acetylaurin wurde zuerst von Graebe und Caro' dargestellt. In der Folge haben Dale und Schorlemmer ${ }^{2}$ dieselbe Verbindung erhalten. Endlich hat auch Zulkowsky ${ }^{3}$ das Acetylaurin in Händen gehabt. Caro und Graebe geben den Schmelzpunkt bei $159-160^{\circ}$ an, während Dale und Schorlemmer denselben bei $168^{\circ}$ fanden. Die von Caro und Graebe angeführten Analysen stimmen mit ihrer Auffassung ganz gut überein, während einige der Analysen von Dale und Schorlemmer im Kohlenstoffgehalte gegen die theoretisch geforderte Zahl eine Differenz von nahezu einem Percent ergeben.

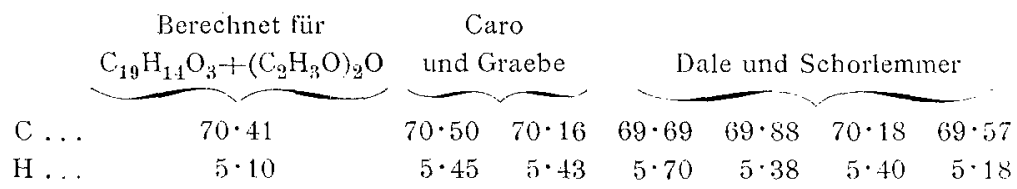

Die von Zulkowsky publicirten Zahlen stimmen mit denen von Caro und Graebe besser überein.

Wir haben nun das Acetylaurin wiederholt dargestellt und dasselbe einer gründlichen Reinigung und Analyse unterworfen. Dabei hatten wir Aurine verschiedener Provenienz zur Verfügung, welche aber allerdings sämmtliche nach dem Verfahren von Zulkowsky dargestellt waren. Herr Prof. Zulkowsky war ausserdem so liebenswürdig, uns zum Vergleich ein reines, von ihm selbst dargestelltes Aurin zu überlassen, wofür wir ihm hiemit unseren besten Dank abstatten.

Die Resultate, die wir beim Studium dieser Verbindungen erhalten haben, sind folgende.

Den Schmelzpunkt des Acetylaurins fanden wir übereinstimmend mit Dale und Schorlemmer bei $168-169^{\circ}$. Bei oftmaligem Umkrystallisiren steigt er noch ein wenig und wird bei $171-172^{\circ}$ ganz constant.

Was unsere Analysenbefunde betrifft, so sind die von uns erhaltenen Zahlen für den Kohlenstoffgehalt noch niederer als alle bis jetzt von den bereits oben citirten Autoren gefundenen

1 Berl. Ber., XI, 1122.

-Ann. der Chemie, 196, S. 84.

3 Ann. der Chemie, 202, S. 191. 
Werthe, so zwar, dass wir gezwungen sind, dem Acetylaurin eine andere Zusammensetzung zuzuschreiben als die bisher geltende.

Die Analysen der Präparate verschiedener Provenienz lieferten nämlich folgende Daten:

I. $0.2754 g$ bei $100^{\circ}$ getrockneter Substanz gaben $0.6973 g$ Kohlensäure und $0 \cdot 1262 g$ Wasser.

II. $0 \cdot 2473 g$ bei $100^{\circ}$ getrockneter Substanz gaben $0 \cdot 6226 g$ Kohlensäure und $0 \cdot 1151 \mathrm{~g}$ Wasser.

III. $0 \cdot 2440 \mathrm{~g}$ bei $100^{\circ}$ getrockneter Substanz gaben $0 \cdot 6183 g$ Kohlensäure und $0.1100 \mathrm{~g}$ Wasser.

IV. $0.2395 g$ bei $100^{\circ}$ getrockneter Substanz gaben $0.6062 \mathrm{~g}$ Kohlensäure und $0 \cdot 1117 g$ Wasser.

V. $0.2350 \mathrm{~g}$ bei $100^{\circ}$ getrockneter Substanz gaben $0.5952 \mathrm{~g}$ Kohlensäure und $0 \cdot 1098 g$ Wasser.

In 100 Theilen:

I. II.

C ....69.05 $68 \cdot 67$

III.

IV. $\mathrm{V}$.

H.... $5 \cdot 09$

$5 \cdot 17$ $69 \cdot 11$ $69 \cdot 03$ $69 \cdot 08$

Aus nachstehender Zusammenstellung ist zu ersehen, dass diese Zahlen viel besser auf die Formel $\mathrm{C}_{\mathbf{2 5}} \mathrm{H}_{\mathbf{2 2}} \mathrm{O}_{7}$ als auf die alte mit $\mathrm{C}_{23} \mathrm{H}_{20} \mathrm{O}_{6}$ übereinstimmen.

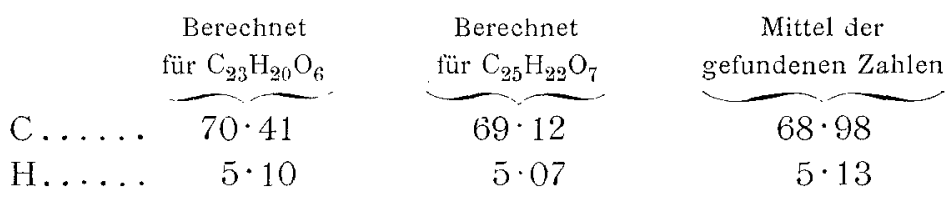

Beide Formeln, $\mathrm{C}_{23} \mathrm{H}_{20} \mathrm{O}_{6}$ und $\mathrm{C}_{25} \mathrm{H}_{22} \mathrm{O}_{7}$, haben das gemeinsam, dass sie sich von einem Körper $\mathrm{C}_{19} \mathrm{H}_{16} \mathrm{O}_{4}$ durch Acetylirung ableiten lassen, während das Aurin die Formel $\mathrm{C}_{19} \mathrm{H}_{14} \mathrm{O}_{3}$ besitzt. Durch den Eintritt zweier Acetylgruppen in das Molekül $\mathrm{C}_{19} \mathrm{H}_{16} \mathrm{O}_{4}$ erhält man die bisher geltende Formel des Acetyiaurins $\mathrm{C}_{23} \mathrm{H}_{20} \mathrm{O}_{6}$, die neue Formel $\mathrm{C}_{25} \mathrm{H}_{22} \mathrm{O}_{7}$ verlangt hingegen den Austausch von drei Wasserstoffatomen gegen Acetylgruppen.

Der Gedanke, durch Bestimmung der Acetyle die neue Formel noch besser als durch Analyse zu stützen, liegt daher 
sehr nahe. Von allen üblichen Methoden zur Acetylbestimmung konnte a priori nur die durch die Destillation in Betracht kommen. Leider gab auch diese nicht sichere Zahlen insofern, als beim Überdestilliren der gebildeten Essigsäure kein scharfes Ende zu erreichen war, so zwar, dass die Flüssigkeit immer noch eine Spur von Ätzkali neutralisirte. Es scheint, als ob sich das Aurin bei der Einwirkung concentrirter Säuren spurenweise unter Bildung saurer flüchtiger Körper zersetzen würde. Wie weit die von uns ausgeführten Acetylbestimmungen trotzdem als für das Vorhandensein dreier Acetylgruppen beweisend angesehen werden können, möge man aus folgender Zusammenstellung schliessen.

Angewandte Substanz: $1 \cdot 2440 \mathrm{~g}$, Titre der Kalilauge $0.0044 g \mathrm{KOH}$.

I. Destillat, $100 \mathrm{~cm}^{3}$, verlangte $70 \cdot 2 \mathrm{~cm}^{3}$ Kalilauge,

\begin{tabular}{|c|c|c|c|c|}
\hline II. & " & $100 \mathrm{~cm}^{3}$, & » & $30 \cdot 0 \mathrm{~cm}^{3}$ \\
\hline III. & $»$ & $100 \mathrm{~cm}^{3}$ & $»$ & $10 \cdot 2 \mathrm{~cm}^{3}$ \\
\hline 1 & $\gg$ & $100 \mathrm{~cm}^{3}$ & » & $4.0 \mathrm{~cm}^{3}$ \\
\hline & " & $250 \mathrm{~cm}^{2}$, & » & $1 \cdot 0 \mathrm{cml}^{3}$ \\
\hline
\end{tabular}

Die Destillate VI, VII und VIII zu je $250 \mathrm{~cm}^{3}$ verlangten $0 \cdot 7,0 \cdot 5$ und $0 \cdot 8 \mathrm{~cm}^{3}$ Kalilauge. Berechnet man die Essigsäure aus den Destillaten I, II, III und IV, so ergibt sich die Zahl $43 \cdot 3$ in Percenten, während die Formel $\mathrm{C}_{25} \mathrm{H}_{22} \mathrm{O}_{7}$ für drei Acetylgruppen $41 \cdot 49 \%$ Essigsäure erfordert. Weitere zwei Versuche, wobei wir unter denselben Verhältnissen ganz ähnliche Resultate erzielten, wollen wir nicht des Näheren anführen. Es soll nun keineswegs behauptet werden, dass đie gefundenen Zahlen absolut beweisend sind, wenn man aber bedenkt, dass die alte Formel für zwei Acetylgruppen $30 \cdot 61 \%$ Essigsäure verlangt, so kann man doch wohl sagen, dass die von uns erhaltenen Zahlen eher für die Richtigkeit der neuen, als für die der alten Formel sprechen.

Die Autoren, die zuerst Acetylaurin dargestellt und ihm folgende Constitutionsformel

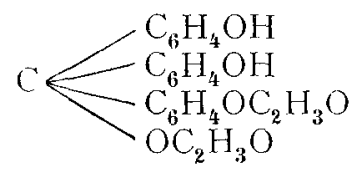


zugeschrieben haben, lösten das Aurin in der Kälte oder höchstens bei Wasserbadtemperatur in Anhydrid auf. Es lag daher für sie immerhin die Möglichkeit vor, dass bei der weiteren Einwirkung von Essigsäure-Anhydrid zumal bei höherer Temperatur auch eine Substitution der Hydroxylwasserstoffe eintreten werde. Dies ist nun nach unseren Erfahrungen nicht der Fall. Wir erhielten das Acetylaurin durch Kochen des Aurins mit Anhydrid und Natriumacetat und konnten uns davon überzeugen, dass durch neuerliche Behandlung des fertigen Acetylaurins mit Anhydrid und Natriumacetat keine weitere Substitution vor sich geht. Auch Acetylchlorid bei $150^{\circ} \mathrm{im}$ Rohr ist ohne Einwirkung auf das Acetylaurin. Man kann daher im Acetylaurin keine freie Hydroxylgruppe annehmen. Damit stimmt auch die Thatsache überein, dass man diese Verbindungen sehr lange mit verdünnter Kalilauge schütteln kann, ohne dass sie sich lösen.

Wir wollen es aber nicht zu bemerken unterlassen, dass auch wir die von Caro und Graebe erwähnte leichte Verseifbarkeit beobachteten. Acetylaurin wurde in der Kälte mit Alkohol befeuchtet, eine concentrirte alkoholische Lösung von Kali hinzugefügt, dann sofort mit viel Wasser verdünnt, wobei sich momentan Alles gelöst hat. Beim Ansäuern mit Essigsäure schied sich alsbald ein rother Körper aus, der nach dem Abfiltriren, Waschen und Trocknen sich hei der Analyse als nahezu reines Aurin erwies. ${ }^{1}$

$0.2530 \mathrm{~g}$ bei $110^{\circ}$ getrockneter Substanz ergaben $0.7221 \mathrm{~g}$ Kohlensäure und $0 \cdot 1110 \mathrm{~g}$ Wasser.

In 100 Theilen:

\begin{tabular}{|c|c|c|}
\hline Gefunden & $\begin{array}{r}\begin{array}{r}\text { Berechnet } \\
\text { für } \mathrm{C}_{19} \mathrm{H}_{14} \mathrm{O}_{3}\end{array} \\
\end{array}$ & $\begin{array}{c}\text { Berechnet für } \\
\mathrm{C}_{19} \mathrm{H}_{13} \mathrm{O}_{3}\left(\mathrm{OC}_{2} \mathrm{H}_{3} \mathrm{O}\right)\end{array}$ \\
\hline $77 \cdot 84$ & $78 \cdot 62$ & $75 \cdot 67$ \\
\hline $4 \cdot 87$ & $4 \cdot 82$ & $5 \cdot 10$ \\
\hline
\end{tabular}

1. Es sei hervorgehoben, dass wir oft aus einem durch Verseifung erhaltenen Aurin das Acetylproduct regenerirt und immer denselben Körper wieder bekommen haben. 
Durch die Versuche von Ackermann ${ }^{1}$ ist die Anwesenheit zweier Hydroxylgruppen im Aurin sicher nachgewiesen. Das Acetylaurin enthält nun, wie bereits oben erwähnt, gar kein freies Hydroxyl mehr. Es muss daher consequenterweise im Acetylderivat von der Formel $\mathrm{C}_{25} \mathrm{H}_{22} \mathrm{O}_{7}$ der Rest des Diacetylaurins $\mathrm{C}_{23} \mathrm{H}_{18} \mathrm{O}_{5}$ präformirt vorhanden sein. Bei Gegenüberstellung dieser beiden Formeln sieht man, dass der Körper $\mathrm{C}_{25} \mathrm{H}_{22} \mathrm{O}_{7}$ angesehen werden könnte als eine lose moleculare Verbindung von Diacetylaurin und Essigsäure. In der That kennt man solche Verbindungen des Aurins mit anorganischen und organischen Säuren, so dass die Annahme einer molecularen Verbindung bei oberflächlicher Betrachtung ganz plausibel erscheint. Geht man aber auf die Sache näher ein, so muss diese Erklärung als sehr unwahrscheinlich fallen gelassen werden. Die Gründe, die dagegen sprechen, sind wesentlich folgende:

Dale und Schorlemmer, ${ }^{2}$ denen wir das Studium der Salze des Aurins verdanken, haben schon bemerkt, dass die Salze des Aurins mit Säuren die rothe Farbe des Aurins besitzen, während die Verbindungen mit den Bisulfiten und dem Essigsäure-Anhydrid farblos sind wie Leukaurin oder Hydrocyanaurin. »Es scheint demnach «, heisst es weiter, »dass die chemische Constitution der Glieder beider Gruppen verschieden ist. « Diese beiden Gruppen unterscheiden sich aber ausserdem auch noch dadurch, dass die Verbindungen mit Säuren ausserordentlich leicht zersetzlich sind, während die anderen Substanzen sich als stabiler erweisen. Salzsäure und Schwefclsäure lassen sich beispielsweise aus ihren Verbindungen mit dem Aurin schon durch kaltes Wasser wegwaschen, während das Acetylaurin selbst mit kochendem Wasser behandelt werden kann, ohne auch nur eine Spur Essigsäure zu liefern. Ebensowenig lässt sich die Essigsäure durch directes Erhitzen entfernen, da selbst die geschmolzene Verbindung noch keine Änderung in ihrer Zusammensetzung erlitten hat. Mit Bezug auf die Farblosigkeit des Acetylaurins könnte man vielleicht

1 Berl. Ber., XVII, S. 1627.

2 Ann. Chem. Pharm., 196, S. 90. 
noch den Einwand erheben, dass ja möglicherweise schon das Diacetylaurin farblos ist, und dass dann selbstverständlich auch die Essigsäureverbindung desselben farblos sein muss. Aber auch dagegen lässt sich ein allerdings nicht ganz vollgiltiges Argument erbringen, indem die von Ackerman n dargestellten Dialkyläther des Tetrabrom- und Tetranitroaurins nicht farblos sind. Immerhin glauben wir im Rechte zu sein, wenn wir der Ansicht von Dale und Schorlemmer beipflichten und daraus den Schluss ziehen, dass das Acetylaurin ähnlich constituirt sein muss wie Leukaurin und Hydrocyanaurin.

Vom obigen Gesichtspunkte aus bieten sich für die Constitution des Acetylaurins zunächst zwei Möglichkeiten dar, welche sich mit Zugrundelegung der Aurinformel von Nietzki folgendermassen entwickeln lassen. Ist das Aurin nach Schema I zusammengesetzt, so könnte das Acetylaurin entweder nach Schema II oder III constituirt sein:

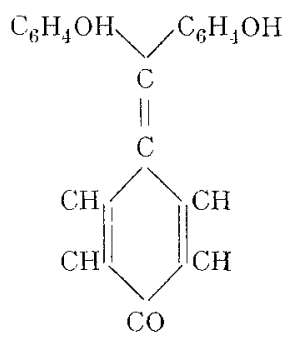

I.

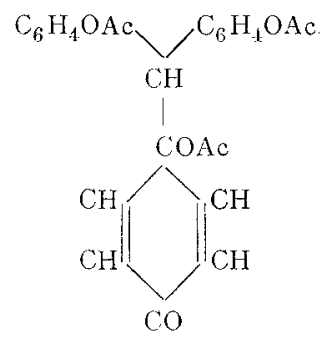

II.

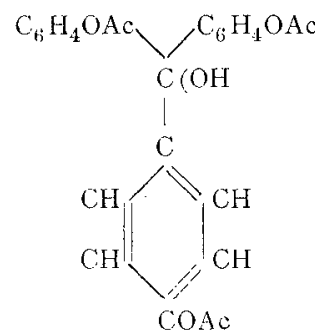

III.

Die Formel Il enthält gar kein freies Hydroxyl mehr und damit wäre erklärt, warum das Acetylaurin in Kali unlöslich ist und wesshalb alle Versuche, eine weitere Acetylgruppe in das Molekül einzuführen, missglückt sind. Andererseits müsste man, um den Parallelismus mit den Leukoverbindungen aufrecht halten zu können, annehmen, dass das Wesentliche an der Bildung der Leukoverbindungen nicht die Umwandlung des Chinonsauerstoffes in die Hydroxylgruppe, sondern die Aufhebung der doppelten Bindung beim Methankohlenstoff ist. Diese Annahme scheint uns aber nach allen in der Literatur vorliegenden Beobachtungen unwahrscheinlich und unstatthaft.

Bei Formel III fällt die oberwähnte Schwierigkeit vollkommen hinweg, da wir es hier in der That mit einem Körper 
zu thun haben, der ganz analog den Leukoverbindungen zusammengesetzt ist. Allein auch diese Formel erscheint uns nicht genügend sicher, weil in derselben ein freies Hydroxyl vorkommt und das gesammte Verhalten des Acetylaurins dafür zu sprechen scheint, dass kein durch Acetyl ersetzbares Wasserstoffatom in dieser Verbindung vorhanden ist. Allerdings sind die Carbinolverbindungen der complicirteren Triphenylmethanderivate nicht so gründlich studirt, um den Schluss als ganz unmöglich erscheinen zu lassen, dass sich einige Carbinolverbindungen beim Acetyliren so verhalten könnten, wie es bei manchen Verbindungen bei der Behandlung mit Kali und Jodalkyl nach den Beobachtungen von Graebe, ${ }^{1}$ Kostanecki ${ }^{2}$ und Herzig ${ }^{3}$ der Fall ist. Besagte Autoren fanden, dass be stimmte Substanzen, welche sicherlich Hydroxylgruppen enthalten, sich sehr schwer oder auch gar nicht alkyliren lassen.

Bevor wir aber in der Discussion weiter schreiten, möchten wir eines Versuches erwähnen, der eine Entscheidung zwischen Formel II und III herbeiführen sollte. Der Gedankengang, der uns dabei geleitet hat, war folgender. Das Chinonsauerstoffatom ist im Aurin durch Wasserstoff leicht in die Hydroxylgruppe umzuwandeln. Nun enthält das Schema II noch die intacte Carbonylgruppe, während sie in der Formel III nicht mehr vorhanden ist.

Man sollte daher denken, dass ein Körper nach Schema II unter Bildung einer neuen Hydroxylgruppe reducirbar wäre, während dies nach Formel III nicht der Fall sein könnte. Acetylaurin wurde daher der Einwirkung von Zinkstaub in essigsaurer Lösung unterworfen, und zwar ging die Einwirkung zuerst in der Kälte vor sich und erst dann wurde die Reaction am Wasserbade zu Ende geführt. Nachdem sich kein Wasserstoff mehr entwickelt hatte, wurde die Reactionsmasse in Wasser eingetragen und die ganze Flüssigkeit mit Äther ausgeschüttelt. Der Äther hinterliess nach dem Abdestilliren eine weisse, krystallinische Masse, welche mehrfach aus absolutem Alkohol umkrystallisirt den constanten Schmelzpunkt $136-137^{\circ}$ besass.

1 Ann. der Chemic, 269, S. 301.

2 Monatshefte für Chomie, XII, S. 320 .

3 Monatshefte für (homic, V, S. 72; IX, S. 548: XII, S. 161 und 177. 
Es ist dies derselbe Schmelzpunkt, den Graebe ${ }^{1}$ für das Triacetylleukaurin angibt und den wir bei derselben Verbindung. zu beobachten bisher oft Gelegenheit hatten. Die mit dem Körper ausgeführten Analysen zeigten, dass hier in der That Triacetylleukaurin vorliegt.

I. $0.2182 \mathrm{~g}$ bei $100^{\circ}$ getrockneter Substanz ergaben $0.5740 g$ Kohlensäure.

II. $0.2227 \mathrm{~g}$ bei $100^{\circ}$ getrockneter Substanz ergaben $0.5873 \mathrm{~g}$ Kohlensäure und $0 \cdot 1077 g$ Wasser.

In 100 Theilen:

$$
\begin{aligned}
& \text { Gefunden } \\
& \text { C. . .71 75 71.92 } \\
& \mathrm{H} \ldots \mathbf{-}^{\mathbf{2}} 5 \cdot 38
\end{aligned}
$$

Für die Constitution des Acetylaurins hat dieser Versuch schon desshalb eine grosse Bedeutung, weil er der schlagendste Beweis für die Anwesenheit von drei Acetylgruppen in demselben ist. Weiterhin ist dadurch sicher festgestellt, dass die drei Acetylgruppen im Acetylaurin dieselbe Stellung haben müssen wie im Acetylleukaurin, und darum erscheinen alle bereits behandelten möglichen Fälle bis auf Schema III höchst unwahrscheinlich und müssen daher fallen gelassen werden.

Wir müssen aber zum Schlusse noch zwei weitere Möglichkeiten in Betracht ziehen, deren Beurtheilung schon desshalb grosse Schwierigkeiten verursacht, weil wir das Verhalten analoger Verbindungen fast gar nicht kennen. Diese beiden möglichen Fälle mögen durch Schema IV und V verdeutlicht werden.

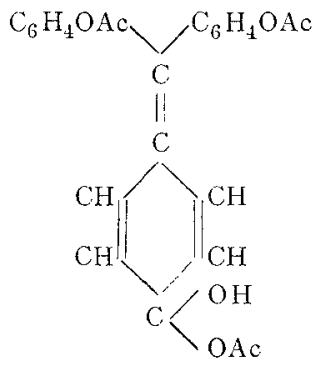

IV.

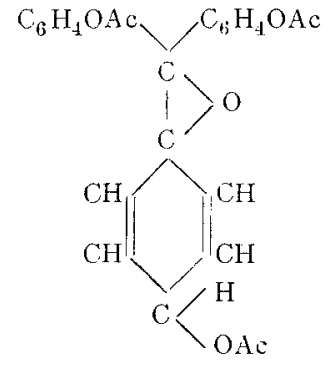

$\mathrm{V}$.

1 Ber\%. Ber, XI, S. 1117 .

2 Die Bestimmung des Wasserstoffes ist verunglückt. 
Formel IV enthält wieder eine freie Hydroxylgruppe, allein wenn wir schon bei der Carbinolformel die Möglichkeit nicht ausgeschlossen haben, dass eine derartige Hydroxylgruppe sich anders gegen Acetyl verhalten könnte wie gewöhnlich, so können wir dies mindestens mit derselben Wahrscheinlichkeit auch hier thun. Ein weiterer Einwand gegen diese Formel wäre der, dass die doppelte Bindung beim Methankohlenstoff nicht aufgehoben erscheint, wodurch der Parallelismus mit den Leukound Hydrocyanverbindungen gestört wäre.

Gegen das Schema V lässt sich gar kein Einwand erheben, da sich dasselbe mit den bisher von uns ermittelten Thatsachen in jeder Beziehung in Einklang bringen lässt. Allerdings konnten wir leider auch keinen directen stricten Beweis für diese Formel erbringen.

Bei der Reduction des Acetylaurins zu Acetylleukaurin müsste sowohl bei IV als wie bei $V$ eine Wanderung des Wasserstoffes vom Chinon- zum Methankohlenstoff und eine subsequente Verschiebung der doppelten Bindungen stattfinden, da das Leukaurin schon mit Rücksicht auf die Hydrocyanverbindungen ${ }^{1}$ folgende Constitution

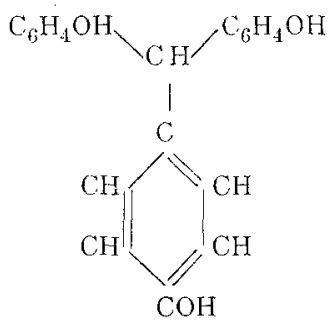

besitzen muss.

So wären wir denn zu drei Formeln des Acetylaurins gelangt, mit welchen wir die einigermassen plausiblen Fälle erschöpft zu haben glauben. Wir sind heute nicht in der Lage, uns mit Bestimmtheit für die eine oder die andere Formel auszusprechen, weil uns dazu weder die Beobachtungen anderer Autoren, noch unsere eigenen Versuche die Mittel liefern.

Wir wollen nun das rein thatsächliche Material, welches wohl kaum modificirt werden wird, hier kurz zusammenstellen.

1 Fischer und Jennings, Berl. Ber., XXVI, S. 2221. 
1. Das Acetylaurin leitet sich nicht vom Aurin $\left(\mathrm{C}_{19} \mathrm{H}_{14} \mathrm{O}_{3}\right)$, sondern von einem Körper $\mathrm{C}_{19} \mathrm{H}_{16} \mathrm{O}_{4}$ ab, liefert aber trotzdem bei der Verseifung Aurin.

2. Bei der Reduction liefert Acetylaurin Triacetylleukaurin. Es enthält demnach drei Acetylgruppen, was auch mit dem Befunde bei der Analyse sehr gut übereinstimmt. Diese drei Acetylgruppen haben dieselbe Stellung wie im Triacetylleukaurin.

3. Eine freie Hydroxylgruppe ist im Acetylaurin nach den gewöhnlichen Methoden nicht nachweisbar.

Nach den in der Literatur vorliegenden Angaben kann man ganz bestimmt voraussetzen, dass ganz dieselben Verhältnisse auch bei den anderen Aurinen vorliegen, und wir gedenken auch die Rosolsäure in den Kreis unserer Betrachtungen zu ziehen. Ausserdem wird unser Streben dahingehen, uns durch das Studium einiger für diesen Zweck besonders geeigneter Carbinolbasen Klarheit über die Functionen des Carbinolhydroxyls bei den complicirten Triphenylmethanderivaten zu verschaffen.

Zum Schlusse möchten wir noch eines Umstandes gedenken, der mit unserer eigentlichen Frage nur in ganz losem Zusammenhange steht. Es ist bereits erwähnt worden, dass Caro und Graebe den Schmelzpunkt des Acetylaurins bei $159-160^{\circ}$ fanden, während die späteren Autoren einen wesentlich höheren Schmelzpunkt beobachten konnten $\left(168-169^{\circ}\right.$, $170-172^{\circ}$ ). Man konnte daher denken, dass Caro und Graebe ein nicht ganz reines Präparat in Händen hatten. Dies ist nicht der Fall. Prof. Graebe, dem wir hiemit unseren Dank abstatten, hatte die Güte, uns eine kleine Probe seines alten Präparates einzusenden, und wir konnten uns überzeugen, dass dasselbe constant bei $157-158^{\circ}$ schmilzt trot $z$ wiederholter Reinigung und mehrfachen Umkrystallisirens. Wir haben dann das alte Präparat nochmals mit Anhydrid und Natriumacetat behandelt, und wieder erhielten wir denselben Körper vom Schmelzpunkt $157-158^{\circ}$. Bei dieser Gelegenheit haben wir die Verbindung 
So oft aus Alkohol umkrystallisirt, dass wir von $3.5 g$ nur $0.2 g$ erhielten, während $3 \cdot 3 g$ in die Laugen gegangen waren, und trotzdem hat sich der Schmelzpunkt nicht geändert. Es ist also kein Zweifel, dass wir es hier mit einem Körper zu thun haben, der mit dem Acetylaurin nicht identisch ist. Die bei der Analyse erhaltenen Zahlen deuten darauf hin, dass ein Methylaurin vorliegt.

I. $0.2642 g$ bei $100^{\circ}$ getrockneter Substanz gaben $0.6735 g$ Kohlensäure und $0 \cdot 1224 \mathrm{~g}$ Wasser.

II. $0.2445 g$ bei $100^{\circ}$ getrockneter Substanz gaben $0.6244 g$ Kohlensäure und $0.1125 \mathrm{~g}$ Wasser.

In 100 Theilen:

\begin{tabular}{|c|c|c|c|}
\hline \multicolumn{2}{|c|}{ Gefunden } & \multirow{2}{*}{$\begin{array}{l}\text { Berechnet } \\
\text { für } \mathrm{C}_{26} \mathrm{H}_{21} \mathrm{O}_{7}\end{array}$} & \multirow{2}{*}{$\begin{array}{l}\text { Berechnet } \\
\text { für } \mathrm{C}_{25} \mathrm{H}_{22} \mathrm{O}_{7}\end{array}$} \\
\hline I. & II. & & \\
\hline $.69 \cdot 52$ & $69 \cdot 65$ & $69 \cdot 64$ & $69 \cdot 12$ \\
\hline$\ldots 5 \cdot 15$ & $5 \cdot 12$ & $5 \cdot 35$ & $5 \cdot 07$ \\
\hline
\end{tabular}

Diese Vermuthung lässt sich aber nicht als ganz sicher hinstellen, da die Differenz in den theoretischen Zahlen eine zu geringe ist. Thatsache ist, dass wir aus einem Aurin, welches durch Verseifung des Acetylproductes vom Schmelzpunkte $170^{\circ}$ entstanden war, das Acetylleuko- und das Acetylhydrocyanderivat dargestellt haben und dass diese beiden Derivate genau den Schmelzpunkt besassen, wie ihn Graebe für die entsprechenden Verbindungen selbst gefunden hat. Es ist daher nicht ganz unmöglich, dass den beiden Autoren gerade beim Acetylaurin ein Methylaurin in die Hände gekommen. Ein Versuch, aus einem kleinen Rest des Acetylaurins von Graebe das Acetylhydrocyanderivat darzustellen, ist uns leider missglückt. 